\title{
Le syndicalisme à l'épreuve de la communication
}

syndicalism to the Test of Communication

\section{Stéphane Olivesi}

\section{(2) OpenEdition}

\section{Journals}

Édition électronique

URL : https://journals.openedition.org/questionsdecommunication/6702

DOI : 10.4000/questionsdecommunication.6702

ISSN : 2259-8901

\section{Éditeur}

Presses universitaires de Lorraine

\section{Édition imprimée}

Date de publication : 1 septembre 2012

Pagination : 269-290

ISBN : $978-2-8143-0120-7$

ISSN : 1633-5961

\section{Référence électronique}

Stéphane Olivesi, «Le syndicalisme à l'épreuve de la communication », Questions de communication [En ligne], 21 | 2012, mis en ligne le 01 septembre 2014, consulté le 27 octobre 2022. URL : http:// journals.openedition.org/questionsdecommunication/6702 ; DOI : https://doi.org/10.4000/ questionsdecommunication.6702

\section{(c) (i) () $९$}

Creative Commons - Attribution - Pas d'Utilisation Commerciale - Pas de Modification 4.0 International - CC BY-NC-ND 4.0

https://creativecommons.org/licenses/by-nc-nd/4.0/ 


\section{$>$ NOTES DE RECHERCHE}

STÉPHANE OLIVESI

Versailles Saint-Quentin Institutions publiques

Université Versailles - Saint-Quentin-en-Yvelines stephane.olivesi@uvsq.fr

\section{LE SYNDICALISME À L'ÉPREUVE DE LA COMMUNICATION}

Résumé. - Souvent caricaturées comme sclérosées ou vieillissantes, parfois dénoncées pour leur conservatisme et leur archaïsme, les organisations syndicales se présentent à l'observateur comme une réalité plus complexe et contrastée qu'on ne veut l'entendre. II est vrai que, confrontées à la communication conçue comme enjeu stratégique, comme terrain symbolique sur lequel se joue l'essentiel des luttes opposant représentants des salariés et employeurs, elles paraissent relativement démunies. L'article se propose d'identifier puis d'expliciter ces problèmes de communication auxquels les organisations syndicales doivent faire face, tant localement pour les syndicats d'entreprise qu'à l'échelon confédéral. S'appuyant sur des données provenant de différentes sources, les analyses tentent de progresser dans la compréhension de ce qui est confusément rangé sous la rubrique « crise du syndicalisme ».

Mots clés. - Syndicalisme, communication, action syndicale, représentation syndicale, organisation syndicale. 
es organisations syndicales auraient perdu la bataille de la communication! C'est du moins la tonalité d'un article paru dans le supplément «Télévisions » du journal Le Monde au terme du conflit social qui, durant l'automne 20 I0, les opposa au gouvernement sur la réforme des retraites. Cet article insistait sur leur incapacité à s'approprier une nouvelle arme de mobilisation massive :leWeb! Cet apparent constat invitait à mesurer leur archaïsme : comment de « vieilles organisations » pourraient-elles s'approprier ces signes éblouissants de notre modernité technique et sociale ? II serait aisé de renvoyer notre pourfendeur des archaïsmes syndicaux dans les cordes du ring journalistique en montrant à quel point son analyse se résume en la rationalisation de quelques préjugés conformes à l'air du temps, le conduisant à méconnaître à la fois la réalité des organisations syndicales, les logiques de mobilisation sociale (Neveu, 2005) et les ressources stratégiques dont elles (ne) disposent (pas) pour agir.

Cela dit, les organisations syndicales sont bel et bien confrontées à des problématiques d'ordre communicationnel, nettement plus complexes que ne le suggère la seule focale du web et des nouvelles formes de sociabilités réticulaires dont il est le théâtre. À divers titres, l'action syndicale relève prioritairement du registre communicationnel, du moins si l'on ne réduit pas ce registre à quelques aspects apparents (tractage, prise de parole publique, négociation) et que l'on intègre l'ensemble des relations que les représentants syndicaux nouent et entretiennent avec les salariés, les sympathisants, les adhérents ainsi qu'avec les employeurs, le patronat et les représentants des autres organisations syndicales. En conséquence, la compréhension des problèmes qui se posent aux confédérations et, surtout localement « à la base », aux syndicats d'entreprise dans leurs actions les plus routinières comme dans les situations de crise ou de conflits passe prioritairement par une analyse de leur capacité à agir communicationnellement, à rendre leurs actions et leurs prises de position légitimes aux regards de leurs différents partenaires de jeu, à mobiliser les salariés, à les faire adhérer à des projets collectifs.

La première des précautions consiste donc à préciser que le fait syndical n'est nullement réductible à une vision tronquée, focalisée essentiellement sur les confédérations et leurs leaders qui, en leur qualité de porte-parole, réussissent encore la prouesse de parvenir à faire croire en l'unité de ces organisations². Contrairement à l'exemple précédent du journaliste qui parle du monde

'L'article publié est rédigé par B. Legendre. II est paru en avant-dernière page dans le supplément «Télévision » du journal Le Monde en date des 21 et 22 novembre 2010.

2 Quand on connait ces organisations, notamment la CGT, on peut trouver cocasse d'entendre des observateurs peu avertis mais faisant autorité dans les médias, affirmer qu'elles s'apparentent à des appareils au fonctionnement centralisé. Nos propres observations résultant de la conception et de l'animation de stages de formation-action (pour des Unions départementales CGT, CGT-FO et CFDT de la région Rhône-Alpes) et les résultats d'enquêtes menées principalement par entretiens à tous les niveaux de ces organisations corroborent largement le constat de F. Piotet (2009a : 243). On trouvera un témoignage particulièrement loquace de ce qu'est la vie syndicale au sein d'une UD (Union départementale) dans F. Piotet (2009b : 8 et sq.) 
syndical comme s'il s'agissait d'une entité homogène et ordonnée, comme si l'on pouvait prendre la partie pour le tout, force est d'appréhender la réalité du fait syndical dans la diversité de ses facettes, pour ne pas dire dans son hétérogénéité, sa complexité et ses contradictions : multiplicité des organisations et de l'offre syndicale, structuration duale (par échelle géographique et par secteurs), fonctionnement et pilotage faiblement centralisé, manque fréquent de coordination en leur sein, autonomie variable de leurs composantes (Andolfatto, Labbé, 2007). Une seconde précaution consiste à décomposer analytiquement les problématiques d'ordre communicationnel qui se posent au monde syndical afin d'en expliciter les termes de la manière la plus précise possible. Cette précaution réhabilite l'ambition d'une approche qui se veut globale tout en évitant à la fois généralisation hâtive et point de vue normatif. Quelles sont donc ces grandes problématiques d'ordre communicationnel ? On peut en dénombrer au moins huit :

I. L'emprise de la communication managériale et, corrélativement, l'effacement de tout contre-modèle d'action ;

2. L'institutionnalisation du dialogue social et la dilution des liens entre représentants syndicaux et salariés ;

3. Les transformations du salariat et la rationalisation de la GRH - Gestion des ressources humaines - dans le sens d'un renforcement de l'individualisation du rapport salarial et d'une disparition des solidarités organiques ;

4. Les faiblesses (sociales) des représentants syndicaux dans le jeu (social) de la communication ;

5. Les carences des organisations syndicales (confédérations) en matière de réflexion stratégique et d'élaboration d'outils de communication destinés aux militants ;

6. L'absence de maîtrise des stratégies de communication et de médiatisation au sein des organisations ;

7. Le manque de ressources stratégiques, symboliques et économiques en matière de communication publique (locale et nationale) ;

8. La très relative sous-utilisation des technologies de l'information et de la communication.

Ces « grandes problématiques »-que l'on souhaite à présent expliciter et discuter - appellent quatre remarques préalables. Par certains traits, elles s'apparentent à des hypothèses explicatives relatives à ce d'aucuns ont pu nommer « crise » du syndicalisme (Mouriaux, 1998 ; Ubbiali, 1993 ; Rosanvallon, 1988). Elles éclairent en tout cas certains facteurs qui expliqueraient partiellement la faiblesse des taux de syndicalisation comparativement à d'autres pays, la désyndicalisation 
dans certains secteurs ou domaines d'activité, mais aussi les difficultés de l'action, au quotidien comme en période de conflit social. Si l'on reprend l'argumentation à charge proposée par deux observateurs rigoureux dans leur ouvrage Toujours moins! Déclin du syndicalisme à la française (Andolfatto, Labbé, 2009), le monde syndical souffrirait de pathologies extrêmement lourdes, au point de desservir les intérêts des salariés, de profiter aux seuls militants professionnels et, surtout, de servir les intérêts de ceux qui le financent et qui sollicitent parfois l'intervention de quelques pompiers du social. On peut supposer qu'une prise en considération plus fine des problématiques communicationnelles est de nature non pas à infirmer ces thèses mais à pondérer sur certains points le diagnostic pour ne pas imputer aux militants syndicaux de terrain les causes de maux dont ils pâtissent. Il faut dire que l'explicitation de ces problématiques ne vise nullement à dégager quelques enseignements définitifs, rapidement transformés en conseils avisés, mais à focaliser l'attention sur des problèmes, à mettre ceux-ci en perspective, à produire de l'intelligibilité au moyen du décentrement-recentrement que l'on souhaite opérer à partir et au moyen de la communication saisie comme enjeu stratégique pour l'action syndicale. Leur présentation suit un ordre qui consiste à placer au premier rang les problèmes liés au terrain, au rapport salarial, à la pratique syndicale d'entreprise et, au second rang, juste après, ceux liés au rôle rempli par les confédérations et à la visibilité de ces organisations à l'échelle des espaces publics locaux et nationaux (François, Neveu, 1999). Cet ordre suggère que les problèmes en matière de syndicalisme et, en conséquence, l'orientation des analyses, se posent d'abord à la base et, non pas, en termes d'orientation syndicale, de décisions confédérales ou de jeu d'appareil comme tendent trop souvent à le suggérer les approches globalisantes et essentialistes précédemment dénoncées. Dernière remarque, chacune de ces problématiques appellerait à elle seule de très longs développements; on se limitera par conséquent à en restituer rapidement les traits essentiels, en tentant de renvoyer aux enquêtes et aux études existantes.

\section{L'emprise de la communication managériale et l'effacement de tout contre-modèle d'action}

Si l'on se place dans la (relativement) longue durée, autrement dit si l'on remonte jusqu'à la fin des années 60, on peut repérer les évolutions du patronat qui le conduiront à transformer radicalement ses conceptions de la gestion du social et à développer des technologies de management répondant aux problèmes posés par la conflictualité inhérente au rapport capital/travail. La rationalisation qui découle du dépassement du modèle fordien se résume en quelques phénomènes saillants : création de la formation professionnelle continue, amélioration des conditions de travail, développement de la participation (management participatif), politique de gestion du personnel (préfigurant la GRH), requalification du dialogue social, etc. Les positions des organisations patronales dans les années 
soixante-dix surprennent rétrospectivement par leur dimension sociale. Positives à divers titres, elles ne doivent pas dissimuler leur raison d'être : modifier la donne sociale afin de répondre aux maux dont pâtissaient les entreprises sous l'angle économique mais aussi social. Turn-over, désimplication, coût de non-qualité, absentéisme et, surtout, très forte conflictualité adossée à une présence syndicale significative, voire massive dans certains cas, définissaient alors le paysage social. L'émergence et le développement de la communication managériale s'inscrivent dans cet horizon. Ils traduisent la capacité des organisations patronales, des dirigeants d'entreprise et de leurs relais, de transformer radicalement leurs positions et leurs méthodes pour (re-)conquérir le terrain perdu ${ }^{3}$. Les années 80 consacreront le triomphe de cette volonté de reconquête symbolique et stratégique. Face à ces évolutions, le monde syndical semble être resté quelque peu figé, intellectuellement et stratégiquement, n'évoluant pas à la même vitesse, subissant le plus souvent ces transformations.

Pour prendre la mesure de ce décalage et de cette évolution à contretemps, une simple observation lexicale suffit (Salem, 1993). La référence à la propagande a longtemps caractérisé la culture syndicale, en particulier celle de la CGT, restant ainsi présente jusqu'à l'orée des années 2000. La permanence du terme ne se résume pas à une simple question lexicale. Elle traduit une opposition de modèles, un conflit interne en termes de valeurs et de conception de l'action, voire de définition du syndicalisme. Ce n'est pas un hasard si l'abandon de toute référence à la propagande a pris autant de temps à s'imposer car, avec elle, disparaissaient des éléments identitaires et une conception du syndicalisme à laquelle de nombreux militants restaient attachés. Globalement, selon des tempos différents, les organisations syndicales sont entrées dans l'ère de la communication à marche forcée devant à la fois renoncer à leur propre conception de l'action (propagande) et s'approprier des armes qui n'avaient pas été initialement forgées pour elles.

À ce titre, leur faiblesse paraît double car cette absence de contre-modèle renvoie tout autant aux contenus de la communication syndicale qu'aux pratiques ellesmêmes. La rhétorique des organisations syndicales qui permettait de donner un sens national voire international à des conflits locaux s'est effacée pour laisser la voie libre au triomphe de l'entreprise comme valeur de société (Sainsaulieu, 1990 ; 1994) et à la rhétorique patronale (concurrence, qualité, compétitivité, etc.). Mais ce phénomène est peut-être secondaire en regard d'autres phénomènes. L'absence de modèle d'action propre aux organisations syndicales se reflète dans l'extrême pauvreté de la réflexion et de l'analyse stratégique. À la base, l'apprentissage de la communication se fait en ordre dispersé. Le plus souvent, il s'opère localement, à partir d'expériences ponctuelles, sans véritable

3 Les actes du colloque des 18 et 19 novembre 2009 «Propagande, information, communication. Cent ans d'expériences de la CGT. De 1895 à nos jours », organisé par l'Institut CGT d'histoire sociale offrent de nombreux témoignages éclairant cette problématique. Accès : http://www.ihs.cgt.fr/spip. php?rubrique66. 
coordination et mutualisation des savoirs. Cette absence de modèle se reflète dans l'hétérogénéité de l'offre de formations en communication, destinées aux représentants syndicaux ainsi que dans les contenus de celles-ci (Olivesi, 20 I I). En définitive, on retrouve des syndicalistes (militants et représentants) souvent désemparés. Le phénomène peut évidemment s'expliquer par l'évocation de facteurs exogènes tels que la faiblesse de la syndicalisation et l'absence de soutien des salariés et, plus essentiel, par l'efficacité des stratégies patronales ou l'environnement économique. En fait, il puise ses racines dans leur incapacité à agir dans des configurations de jeu qui les dépassent en l'absence de modèles, de cadres cognitifs et pratiques sur lesquels s'appuyer pour fonder leur action tant à l'égard des salariés que des directions d'entreprise, comme si l'institution syndicale à laquelle ils appartiennent les avait laissés désarmés face à un adversaire qui n'a même plus à livrer bataille.

\section{L'institutionnalisation du dialogue social et la dilution des liens entre représentants syndicaux et salariés}

La seconde problématique renvoie aux liens fortement distendus et parfois rompus entre représentants syndicaux et salariés. Les explications ou tentatives d'explications abondent. Longtemps, le monopole de la représentativité (détenu par la CGT, la CFDT, la CGT-FO, la CFTC et la CGC) fut tenu pour une des principales causes de ce phénomène. Trop souvent, ces représentants ne se souciaient guère de leur audience auprès des salariés. Le dispositif d'ARTT (aménagement et réduction du temps de travail) dans le cadre de la première loi Aubry (I3 juin 1998) avait révélé l'ampleur du problème, c'est-à-dire la très discutable représentativité des représentants « officiels », voire en de nombreux cas au sein des PME leur absence pure et simple (Olivesi, 200l). Et depuis longtemps, de nombreux observateurs, sans même évoquer des acteurs du monde syndical n'appartenant pas au « club des cinq » et subissant les effets de cette situation, avaient dénoncé ce monopole à un point tel que les organisations syndicales « représentatives » qui avaient le moins intérêt à ce qu'une réforme modifie la donne finirent par reconnaître la nécessité de réformer la définition de la représentativité. La situation résultant de la loi de rénovation de la démocratie sociale du 20 août 2008 laisse entrevoir un changement d'attitude chez un nombre significatif d'acteurs locaux et nationaux, plus soucieux de se tourner vers les salariés et de tenter tardivement de renouer des liens avec ceux-ci4.

\footnotetext{
${ }^{4}$ Au fondement de cette étude, des entretiens menés auprès de responsables d'UD (unions départementales CGT et CGT-FO) et d'URI (Unions régionales interprofessionnelles CFDT) ainsi qu'auprès d'acteurs fédéraux et confédéraux. Par conséquent, l'enquête et le contenu des entretiens portaient essentiellement sur l'offre de formation en communication mais, au sein de chacun d'eux, étaient évoqués les changements résultant de la réforme de la représentativité afin d'apprécier dans quelle
} 
Une autre cause résiderait dans les conséquences indirectes des lois Auroux, notamment celle du 28 octobre 1982 relative au développement des institutions représentatives du personnel. Cette explication se résume en ces termes: la volonté d'institutionnaliser le dialogue social et de créer une sorte de démocratie d'entreprise en renforçant le pouvoir des institutions représentatives aurait en quelque sorte dévoyé l'action syndicale en transformant les représentants des salariés en partenaires privilégiés d'un nouveau jeu formel les associant aux directions. Dans les grandes entreprises, ces représentants auraient ainsi tourné le dos à la base sous l'effet de l'affirmation de leur rôle, de la concentration des mandats, de l'extension de leurs compétences et, surtout, des stratégies de directions préférant enliser ceux-ci dans d'interminables réunions et des débats techniques plutôt que de les laisser militer sur le « terrain ». Le renforcement de la judiciarisation de l'activité syndicale n'a pu qu'amplifier le phénomène (Willemez, 2003/4). Dans les entreprises de taille plus modeste, les représentants auraient surtout enregistré un accroissement de leurs tâches en raison du manque de compétences techniques, pourtant requises pour suivre certains dossiers et, surtout, pour coordonner correctement la concertation avec les salariés. Enfin, le patronat aurait développé des stratégies de gestion des relations sociales à partir de cette redéfinition du jeu pour contenir, voire éliminer en force ou en douceur les représentants les plus virulents (intimidation, endiguement, promotion, etc.), gratifier les plus dociles et diviser les salariés en toutes circonstances.

Au sein des entreprises, mais aussi au sein des organisations syndicales (unions interprofessionnelles ou fédérations), les militants les plus aguerris, les plus opérationnels sous l'angle de la coordination et de l'animation de l'action syndicale sont les premiers promus et, simultanément, ceux qui sont portés à s'éloigner des salariés en raison des mandats divers et variés qui leur incombent, quand ils ne deviennent pas permanents. À ce titre, l'institutionnalisation du monde syndical génère de redoutables effets puisqu'elle revient à éloigner du terrain des luttes sociales ceux qui devraient être au premier rang. Ainsi, mieux que ne pourrait le faire le patronat, la division du travail syndical contribue-t-elle à mettre à distance les représentants de ceux qu'ils représentent. Une autre hypothèse explicative, avancée à diverses reprises par Dominique Andolfatto et Dominique Labbé (1997:59-60), réside dans le repli des organisations syndicales sur elles-mêmes. II est vrai que l'importance des frais de fonctionnement et, corrélativement, la baisse constante des sommes investies dans les actions de communication témoigne de ce phénomène :

«Les dépenses administratives absorbent partout plus des deux tiers du budget ordinaire. Au début des années 50, elles en consommaient moitié moins [...]. La croissance (des frais de personnels et du financement des locaux) traduit le gonflement de l'appareil central et sa tendance à fonctionner pour lui-même. En contrepartie, les dépenses de "propagande" ont été considérablement réduites. L'information syndicale, la diffusion des brochures, les affiches,

mesure cette réforme impliquait le développement de la formation en communication pour mieux préparer les représentants aux échéances électorales. 
etc. constituent aujourd'hui moins de $10 \%$ des dépenses ordinaires contre un tiers il y a un demi-siècle ».

Ces hypothèses explicatives n'épuisent cependant pas la palette des facteurs ayant contribué à la dilution des liens entre représentants syndicaux et salariés. En effet, il faut considérer la difficulté des représentants à s'adresser aux salariés et, plus encore, à les mobiliser. Certes, on peut une nouvelle fois se tourner vers des facteurs conjoncturels exogènes (situation de l'emploi, généralisation des contrats à durée déterminée, individualisation des carrières, restructurations, nouvelles formes de management, etc.), mais il faut surtout prendre en compte les difficultés que rencontrent les représentants pour se rendre crédibles et légitimes aux yeux des autres salariés. Une approche compréhensive de leur vécu et de leur propre perception de l'action syndicale éclaire ce point. D'après leurs témoignages ${ }^{5}$, les salariés développeraient souvent des sentiments oscillant entre indifférence, méfiance, voire hostilité à leur égard. Le plus souvent, ils ne s'adresseraient à eux qu'en situation de besoin ou d'urgence pour que leurs droits soient respectés et défendus à titre individuel. Ils seraient donc perçus non pas comme des défenseurs d'intérêts collectifs, comme des coordinateurs de cette défense et encore moins comme les défenseurs de valeurs, de traditions ou de conceptions du monde social, mais comme des sortes de relais institutionnels, au sein d'un monde désenchanté, dont on peut solliciter les services en dernier ressort. On ne sait quelle est la justesse de ce ressenti, ni quelle est son extension exacte. II façonne en tout cas le vécu de nombreux représentants syndicaux qui, mis en situation d'analyse et de formalisation de leur stratégie de communication à destination des salariés livrent ces perceptions et, par conséquent, leur difficulté à s'adresser aux salariés et à être entendus d'eux. L'exemple du tractage est, à ce titre, exemplaire. Souvent, les militants évoquent le problème que leur pose le très faible impact de ces tracts, rarement reçus, peu lus, peuplant trop rapidement à leur goût, les poubelles.

Le souci des syndicalistes de se former à la communication pour dépasser ce genre de difficultés témoigne de leur perception de ce handicap et de leur difficulté à échapper aux formes de stigmatisation dont ils font l'objet de la part de leurs collègues salariés. Enclins à les chahuter, ces derniers les identifieraient souvent comme des acteurs préoccupés principalement par la jouissance de leurs heures de délégation et par leur statut de salariés protégés. II n'est d'ailleurs pas rare d'enregistrer qu'entre eux, ces mêmes syndicalistes ironisent sur l'usage de ces prérogatives incomprises des autres salariés. L'idéaltype du défenseur des droits des salariés s'estomperait donc derrière celui du « planqué », soucieux de s'émanciper du labeur et de jouir des petits avantages que lui vaut sa collaboration

\footnotetext{
${ }^{5}$ Ces témoignages ont été recueillis dans le cadre de l'animation des stages de formation-action précédemment évoqués. À divers moments, les militants (élus $\mathrm{CE} / \mathrm{CHSCT}$, DP, DS, etc.) sont conduits à évoquer puis à objectiver leurs rapports aux salariés en explicitant tous les indices dont ils disposent leur permettant d'apprécier l'image que les salariés ont d'eux, de l'organisation syndicale à laquelle ils appartiennent, voire du syndicalisme.
} 
institutionnelle au dialogue social avec la direction. Cette dépréciation dont de nombreux représentants pâtissent paraît varier selon les secteurs d'activité et les organisations syndicales ${ }^{6}$. Elle s'apparente à un symptôme de la dilution des liens entre représentants et salariés. Elle s'explique donc par la convergence et l'accumulation d'effets de différentes natures : le contexte social général, les doutes persistants sur l'efficacité, voire la probité des représentants, les doutes non moins lourds relatifs à l'efficacité de toute action syndicale, l'individualisation croissante du rapport au travail, mais aussi la saturation de l'information qui rend inaudible le message syndical, l'image des confédérations et de l'action syndicale en général, l'absence de formats, de supports, de messages originaux susceptibles de focaliser l'attention, etc.

\section{Les transformations du salariat et la rationalisation de la GRH}

La communication telle qu'elle se noue ou ne se noue pas entre représentants et salariés n'est pas indifférente aux statuts de ceux-ci, à la division du travail à l'échelle de la société et à l'intérieur de l'entreprise, aux dispositions contractuelles qui les lient à l'employeur, aux principes de management qui conditionnent leur implication... D'ailleurs, on peut lire les transformations du salariat - diminution de la population ouvrière et redéfinition de celle-ci (Schwartz, 2002 ; Beaud, Pialoux, 2004 ; Terrail, 1990) -, tertiarisation de l'ensemble de l'économie pardelà le développement du seul secteur tertiaire, effacement de la qualification au profit de la compétence, logique d'individualisation de la rémunération, acculturation managériale...), comme autant de facteurs de nature à dissiper les solidarités organiques sur lesquelles reposait en partie le militantisme syndical. Ces solidarités supposaient des adhésions « extrodéterminées », fondées sur le sentiment d'appartenance au groupe mais aussi sur la contrainte que pouvait exercer le collectif sur l'individu, l'incitant à se syndiquer (Andolfatto, Labbé, $2007: 17)^{7}$.

\footnotetext{
6 II n'est malheureusement pas possible de gagner en précision sur ce point si ce n'est à envisager une enquête quantitative, difficilement réalisable, qui permettrait à la fois de mesurer en quoi la dépréciation des représentants affecte différemment les syndicats, les secteurs d'activité, les types d'entreprise, etc. Nos observations « locales » et ponctuelles conduisent en tout cas à supposer que certaines organisations syndicales sont plus affectées que d'autres par ce phénomène.

7 L'analyse qualitative du passage à la syndicalisation et les essais d'objectivation des éléments déterminant le choix d'une organisation syndicale, basés sur des récits de présentation de soi, conduisent à prendre quelques distances avec les typologies relatives aux motivations individuelles d'adhésion. L'adhésion paraît souvent mêler utilitarisme de conjoncture, introdétermination liée à l'histoire personnelle (familiale et sociale) et extrodétermination résultant du contexte général sans que l'on puisse réellement préciser quels facteurs explicatifs l'emportent (Olivesi, à paraitre). De plus, les explications mono-causales livrées par les acteurs eux-mêmes, expliquant par exemple qu'ils se sont syndiqués en fonction d'un lien amical, d'une sensibilité héritée d'une mère ou d'un oncle, d'un contexte social particulier relèvent de reconstructions a posteriori largement déterminées à la fois
} 
Dans un contexte d'effacement de ce type de solidarité,il devient plus difficile pour les représentants syndicaux de tenir un discours audible par un grand nombre de salariés, plus portés à ne considérer que leur intérêt particulier pris isolément ou, au mieux, celui de la sous-catégorie dans laquelle ils se rangent qu'à entrevoir sous la forme de l'intérêt collectif leur propre intérêt. Pour reprendre le lexique du marketing, le ciblage s'impose à partir du moment où les destinataires de la communication syndicale ont des points de vue trop divergents résultant de leur différence de position, d'appartenance, de trajectoire, de rapport au travail. Dans ce contexte, développer une communication au-dessus des intérêts individuels fait encourir aux représentants syndicaux le risque d'être inaudible ; inversement, développer une communication ciblée revient à renforcer les clivages existants, les divisions et à écarter plus encore la possibilité de l'action collective. On mesure la difficulté pour des représentants syndicaux de développer une communication maîtrisée, rationalisée en de tels contextes. Quand ils s'interrogent, quand ils cherchent à être entendus par les salariés et, pour ce faire, à améliorer l'efficacité de leur communication, ils se focalisent parfois sur des détails formels (bonne présentation d'un tract, clarté dans la formulation du message, attractivité de la démarche, etc.), mais ils ressentent souvent qu'au-delà de ces aspects pratiques, ce qui est en jeu plus fondamentalement, c'est la possibilité même de communiquer dans un tel environnement professionnel. Ce ressenti entraîne parfois chez eux des attitudes que l'on pourrait juger sectaire (défense exclusive de tel ou tel groupe et des intérêts de ses membres, etc.) ou arbitraire (stratégies de repli sur le groupe des adhérents ou des sympathisants, voire indifférence). Les apories de la communication syndicale liées à la limitation des possibilités d'action collective et au renforcement des comportements individualistes paraissent découler des évolutions du salariat et du droit du travail. Le développement de ce dernier s'est opéré en effet dans le sens d'une individualisation du rapport salarial au sens où les droits collectifs résultant de rapports de force généraux entre employeurs et groupements de salariés se sont en partie effacés aux profits de droits octroyés à des individus contractants. Cette évolution se mesure à l'aune du recours croissant aux prud'hommes dans les arbitrages salariés/employeurs. Elle se reflète aussi dans la juridicisation des rapports sociaux, le droit étant une arme stratégique omniprésente pour les acteurs dans leur vie quotidienne au travail. Ces évolutions dotent les salariés pourvus de compétences et de formations de haut niveau, de ressources stratégiques appréciables qui les dispensent en grande partie de recourir à des représentants syndicaux dans la défense de leurs droits ; elles tendent à priver les autres de ressources en les abandonnant aux méandres d'un droit du travail réservés aux juristes aguerris ou, en tout cas, à ceux qui peuvent les solliciter. L'individualisation du rapport au travail et l'évolution du droit induisent une perte de sens de l'action collective. Pour les salariés, se référer aux représentants syndicaux ne signifie plus adhérer à des projets collectifs, si ce n'est en certaines circonstances extrêmes (fermetures de site, délocalisations,

par le contexte social de l'évocation et par la volonté de produire, à la fois pour soi comme pour ses interlocuteurs, une représentation de soi cohérente. 
licenciements massifs, etc.) qui peuvent encore fédérer au-delà des oppositions et générer des contextes sociaux au sein desquels les salariés redeviennent perméables à la communication syndicale parce qu'ils attendent et demandent une information fiable, vitale pour eux. De manière générale, les représentants syndicaux, s'ils se risquent à répondre mécaniquement aux attentes des salariés, sont érigés en interlocuteurs-conseils auquel on s'adresse uniquement en cas de difficultés personnelles. Évidemment, l'enjeu est de laisser venir à eux les passagers clandestins pour tenter de les faire adhérer dans un second temps.

\section{Les faiblesses des représentants syndicaux dans le jeu social de la communication}

Les représentants syndicaux ont souvent dû se plier aux libertés qui leur étaient octroyées, libertés formelles dont ils ont rarement pu tirer profit en raison d'un déficit de ressources stratégiques mais aussi de compétences techniques et sociales (Contrepois, 2003). En matière de communication, un même constat s'impose. Les représentants syndicaux dont on pourrait supposer à tort qu'ils s'engagent dans l'action au sein de leur organisation en raison de quelques prédispositions ou de facilités qui les conduisent à prendre la parole, à s'exprimer, à dialoguer, à négocier sans trop de difficultés s'avèrent peu formés pour ce genre d'action symbolique. Ce n'est donc pas en raison de prédispositions qu'ils se destinent à devenir représentants, même si l'on peut supposer que certains d'entre eux ont pu en acquérir le goût au fil d'expériences antérieures ou de sollicitations gratifiantes provenant de leur entourage professionnel (Olivesi, à paraître). Des épreuves telles que la prise de parole publique, la mise en scène de soi dans des situations de la vie professionnelle ou la négociation sociale requièrent la maîtrise d'une forme de violence symbolique. Elles supposent l'acquisition de savoir-faire et de dispositions qui ne sont en rien innés. Une des grandes faiblesses des représentants syndicaux dans leur action locale quotidienne comme dans les situations extraordinaires de la vie de leurs organisations réside précisément dans leur difficulté à jouer le jeu social de la communication, face à d'autres acteurs dotés de ressources dont ils sont manifestement dépourvus. Cette absence ou ce manque relatif de ressources symboliques s'explique d'abord par leur formation initiale et leur trajectoire. Les représentants syndicaux se présentent très rarement comme des cadres ou des agents ayant suivi des formations académiques généralistes, encore moins des formations en droit, en économie ou dans les sciences humaines et sociales. La plus grande partie d'entre eux, y compris quand ils parviennent à progresser professionnellement, ont au mieux suivi des formations techniques. Or plus le dialogue social revêt une forme institutionnalisée, plus son contenu et sa forme s'avèrent élaborés, plus en conséquence la communication se complexifie, et plus ces acteurs manquent des ressources nécessaires pour jouer un jeu qui suppose des compétences particulières. Encore faut-il ajouter que ces compétences sont précisément celles 
que le système éducatif sanctionne. Pour ces agents, souvent rejetés du système éducatif, ces compétences s'apparentent à de redoutables armes de domination symbolique (Bourdieu, 1977) car elles réactivent la mémoire de l'échec jouant sur leur propre inconscient. La sanction symbolique qu'ils ont subie s'avère d'autant plus douloureuse et marquante que leur destinée en a été scellée. Certains récits de vie, développés et recueillis dans le cadre d'un dispositif d'autoscopie (Olivesi, à paraître), conduisent de l'échec scolaire jusqu'à l'adhésion syndicale comme si, inconsciemment, l'engagement syndical relevait d'une sorte de révolte contre cette injustice première au double sens où elle vient en premier et où elle détermine tout le reste. C'est dire que confrontés à des normes qui sont indissociablement celles de l'excellence académique et de la domination sociale qu'ils subissent, ils s'avèrent doublement démunis. Objectivement, ils ne disposent pas des savoirs formels et informels de leurs interlocuteurs, cadres et représentants des directions ; subjectivement, sous l'effet de leur assujettissement, ils se vivent comme incompétents au sens où les compétences que requiert le jeu social de la communication sont précisément celles dont ils manquent et pour lesquelles ils ont été sanctionnés négativement. Les représentants syndicaux ressentent fortement cette carence. Elle se traduit par des demandes de formation en communication qui peuvent paraître peu cohérentes au premier abord (Olivesi, 20l I). Ces demandes se concentrent souvent sur les techniques d'expression mais aussi sur d'hypothétiques astuces qui permettraient de compenser leurs lacunes et d'agir efficacement comme par magie. II est vrai qu'il existe une sorte de magie propre à l'efficacité des acteurs dominants. Mais les principes de cette magie ne résident pas, si ce n'est illusoirement, dans des savoir-faire éprouvés ou dans des techniques de manipulation. Elle traduit bien davantage la prédisposition des dominés à reconnaître la légitimité de la domination qu'ils subissent en prêtant aux dominants un Pouvoir dont ils seraient dépourvus. La magie de la violence symbolique (Bourdieu, 1977) est d'inscrire durablement dans les structures mentales la domination au point de faire des dominés les principaux artisans de cette même domination. Ces derniers ne s'émancipent pas de l'être-dominé auquel ils s'identifient durablement.

L'analyse de ces besoins de formation laisse entrevoir une inégalité difficilement surmontable face à un jeu social dont les règles sont établies pour asseoir la domination et lui conférer son assise symbolique. Mais à trop insister sur ces facteurs liés aux seules inégalités de ressources des représentants syndicaux, on risque de négliger qu'issus de la base pour la plupart, ils présentent des caractéristiques sociales qui leur garantissent aussi une forme d'adéquation avec ceux qu'ils représentent et, inversement, d'inadéquation sociale avec ceux auxquels ils s'opposent. II n'y aurait donc là rien de problématique, si ce n'est que le jeu social de la communication se joue avec les règles de ceux auxquels ils s'opposent. 


\section{Les carences des organisations syndicales en matière de réflexion stratégique et d'élaboration $d^{\prime}$ 'outils de communication destinés aux militants}

Les réponses apportées aux besoins en matière de formation à la communication des représentants syndicaux se concentrent dans les offres de stages proposées soit par les UD (Unions départementales pour la CGT et la CGT-FO) et les URI (Unions régionales interprofessionnelles pour la CFDT), soit par les fédérations (Olivesi, 20 I I). Ces offres sont développées par certaines fédérations, ignorées par d'autres pour des raisons qui ne sont pas qu'économiques ou matérielles. Dans certains départements et certaines régions, une telle offre de formation en communication est très développée ; en d'autres lieux, elle est absente. Un même constat vaut pour les fédérations, invitant à tirer plusieurs enseignements. En premier lieu, on peut observer l'absence de politique concertée et coordonnée de formation à l'échelle des confédérations. II n'y a là rien de propre au secteur de la formation. La double structuration syndicale, par strates géographiques interprofessionnelles et par secteurs d'activité, ne renforce pas la lisibilité d'une offre qui se dessine avant tout à l'échelon local selon des facteurs conjoncturels et des stratégies d'acteurs relativement aléatoires. Les observateurs peu avertis imaginent des organisations très centralisées, le sommet dictant sa conduite à la base. À la vérité, les organisations syndicales, en particulier la CGT et, dans une moindre mesure, la CFDT et la CGT-FO pour des raisons et selon des modalités très différentes d'ailleurs, octroient à leurs composantes (fédérations et unions interprofessionnelles) une très large autonomie au point de donner parfois l'impression qu'il n'y a aucune coordination entre les acteurs en leur sein et, pire, aucun échange d'informations permettant de capitaliser des savoir-faire et de mutualiser ce qu'en management, on appelle de « bonnes pratiques ». Dans ce contexte, un représentant syndical bénéficiera ou ne bénéficiera pas d'une formation en communication non pas selon ses besoins mais selon des circonstances qui lui échappent en grande partie et qui semblent d'ailleurs échapper à tous les acteurs concernés. Un deuxième enseignement concerne la communication et la politique de formation à la communication. Si les pratiques à l'échelon local s'avèrent si hétérogènes, c'est non seulement parce que le travail de coordination et d'homogénéisation de l'offre n'a pas lieu mais parce que, comme on l'a déjà précédemment entrevu, les confédérations n'ont jamais réellement pris la mesure de cette problématique et n'ont jamais réellement cherché à élaborer des modèles d'action adaptés aux spécificités de l'action locale de manière à permettre aux représentants de contrebalancer les stratégies managériales. De trop rares documents témoignent de l'esquisse en trompe l'œil d'une prise en compte de ce problème. En témoigne un unique fascicule diffusé dans les années 90 par la CFDT qui reprenait sous une forme appauvrie des recettes parmi les plus éculées de la formation en communication (CFDT, 1994). En témoignent également les actes d'un séminaire de la CGT (ISERES, 2000) dont le caractère très universitaire (dans la forme et dans le fond) témoigne 
d'une véritable incapacité à se mettre en lieu et place des syndicalistes de terrain pour saisir leurs besoins opérationnels et répondre à leurs attentes. Cette vacuité intellectuelle et stratégique des confédérations sur les questions de communication laisse perplexe quand on sait que l'essentiel de l'action des représentants syndicaux se joue pourtant sur ce terrain. Elle vient à l'appui de la thèse d'une institutionnalisation des organisations syndicales, synonyme de repli sur elles-mêmes et de désinvestissement de l'action syndicale de terrain.

\section{L'absence de maîtrise des stratégies de communication et de médiatisation au sein des organisations}

Au sein des organisations, en l'absence de modèle d'action clairement établi, les représentants syndicaux subissent la communication managériale. Sans aller jusqu'à imaginer des salariés dépourvus de sens critique, adhérents sans restriction à l'information patronale, force est d'observer que très souvent celle-ci s'impose à eux sans qu'ils aient la possibilité de s'y soustraire ou de puiser à d'autres sources leurs représentations du travail et de l'entreprise. Cette emprise s'explique par la mobilisation de moyens sans commune mesure mais aussi par le déploiement de dispositifs managériaux et le développement de technologies de communication visant à disqualifier tout autre discours pour instaurer ce que l'on pourrait nommer en référence à Gramsci « une hégémonie d'entreprise ». Particulièrement significatif est, à ce titre, le développement et la généralisation des baromètres d'opinion ${ }^{8}$. Une réaction spontanée consisterait à déployer une critique inspirée par des travaux tels que ceux de Pierre Bourdieu et montrer que les postulats sur lesquels reposent ces pratiques sondagières sont largement discutables (Bourdieu, 1973 ; Lehingue, 2007). Mais, dans ce cas, peut-être est-ce donner trop de dignité à un objet qui ne s'embarrasse pas de considérations méthodologiques et, moins encore, de problèmes épistémologiques, comme on peut le relever en considérant une multitude de cas faisant fi de la plus élémentaire prudence en matière d'administration des questionnaires, de respect de l'anonymat des personnes interrogées, de prise en compte des nonréponses, d'interprétation des données, d'élaboration des questions posées, etc. S'ils donnent l'illusion de la rigueur et puisent leur crédibilité dans l'adulation de la quantification, largement répandue sous l'effet de la culture gestionnaire, ces baromètres d'opinion ne sont pas des outils de connaissance - ont-il un jour prétendu l'être? - au sens où leurs promoteurs chercheraient à connaître la réalité des opinions des salariés. Ils s'imposent d'abord comme des instruments de communication et d'action, répondant à une double finalité : renforcer et

\footnotetext{
${ }^{8}$ On s'appuie sur une enquête réalisée à partir de quatre séries d'entretiens administrés auprès de consultants, de responsables RH/Com, de représentants syndicaux et de salariés. Cette enquête fera l'objet d'une publication restituant avec plus de précisions les résultats obtenus.
} 
étayer l'imposition de systèmes de représentations, conformes aux intérêts des directions d'entreprise ; neutraliser préventivement l'émergence de tout contrediscours promouvant des représentations discordantes de la réalité sociale au sein des organisations.

Quelle crédibilité et quel poids peut avoir la parole d'un représentant syndical quand il dénonce des conditions de travail que $80 \%$ ou $90 \%$ des salariés sont censés trouver satisfaisantes? On mesure l'opérationnalité stratégique de ces baromètres d'opinion dans la neutralisation des représentants syndicaux; ceux-ci s'avèrent dépossédés de toute ressource argumentative. Ils doivent discourir moins en fonction des orientations de leur organisation syndicale ou des préoccupations des salariés qu'ils représentent, que selon les opinions construites dans le cadre de ces dispositifs. Conçu et utilisé comme un outil de communication interne, le baromètre est censé livrer la réalité de l'opinion des salariés bien mieux que ne peut le faire un acteur, jouant un rôle de médiateur, ne représentant qu'imparfaitement une partie des salariés. C'est dire que, dépossédées de leur légitimité, la parole et l'action du représentant syndical font l'objet d'une double neutralisation. D'abord, son discours se doit de négocier avec la diffusion massive et l'imposition de représentations conditionnant préventivement le dialogue social ; ensuite, en situation de négociation, il fait l'épreuve de son illégitimité et de son impuissance à contredire ce qui a valeur de vérité collective « scientifiquement » construite. Les utilisateurs des baromètres d'opinion parviennent à réaliser cette opération magique qui consiste non seulement à donner l'illusion que les directions se préoccupent des opinions des salariés, à conditionner le regard que les salariés eux-mêmes portent sur leur propre environnement professionnel, mais à faire passer les représentations promues par le management pour des représentations neutres, conformes à la réalité. Ils permettent ainsi au management d'asseoir son action sur un environnement symbolique et cognitif rationalisé qui lui est favorable. S'opposer à ces représentations miraculeusement « neutres » qui nourrissent la communication interne reviendrait en effet pour les représentants syndicaux à assumer le vilain rôle de ceux qui défendent des intérêts particuliers contre l'intérêt général, développent un point de vue sectaire, méprisent la démocratie. La diffusion massive de ces technologies de communication laisse une nouvelle fois entrevoir l'absence de réflexion stratégique des organisations syndicales (confédérations). C'était déjà le cas avec l'extension de toutes les pratiques participatives qui furent accueillies de manière contradictoire par les organisations syndicales, oscillant entre la satisfaction de voir les salariés enfin consultés et associés aux décisions locales et, par ailleurs, la crainte d'être dépossédé de leur base sous l'effet de l'adhésion de ces mêmes salariés aux orientations managériales. On peut même considérer qu'avec les baromètres, les organisations syndicales se trouvent en partie prises à leur propre piège puisqu'elles recourent à leur tour à des sondages. Tous les ans, la CGT confie à l'institut CSA, plus précisément au Département Politique-Opinion, une enquête annuelle, intitulée « baromètre d'image des syndicats et de la CGT auprès des français » qu'elle publie ensuite dans Le Peuple et la Vie ouvrière. Le recours 
aux instituts de sondages et à leurs services n'appellerait pas de commentaires particuliers s'il n'attestait de l'impuissance de ces mêmes organisations à se vivre en adéquation avec les salariés, avec leurs adhérents, à comprendre leurs attentes et leurs besoins autrement que sous cette forme biaisée.

\section{Le manque de ressources stratégiques, symboliques et économiques en matière de communication publique (locale et nationale)}

Aux problèmes liés à la médiatisation de l'information au sein des entreprises s'ajoutent ceux inhérents aux relations avec les médias et à la publicité de l'action syndicale. Là, il importe de distinguer confédérations et acteurs locaux (voire fédéraux en certaines circonstances) car les problèmes se posent en des termes différents. Si l'on considère d'abord la population des représentants syndicaux (DS - délégué syndical -, élus CE - comité d'entreprise/CHSCT - comité d'hygiène, de sécurité et des conditions de travail -, voire secrétaire d'UL - union locale - ou d'UD - union départementale), c'est-à-dire la population de ceux qui, dans le cadre de leurs fonctions, peuvent être conduit à s'exprimer dans les médias ou à être en contact avec des journalistes, on peut observer que, dans leur grande majorité, leurs représentations de cet univers s'avèrent souvent biaisées. Pour eux, si l'on force le trait, « le » journaliste se résume souvent au présentateur du JT. On l'imagine bourgeois et conservateur. En tout cas, le regard qu'il est censé porter sur le monde syndical ne peut qu'être négatif. Le localier et le pigiste qui sont pourtant des interlocuteurs naturels de ces acteurs, parce que les échos de leurs actions ne dépassent guère les frontières du département et parce que le social (Lévêque, 2000) n'est pas le genre le plus prisé dans la presse (locale et nationale, écrite et audiovisuelle), n'ont paradoxalement que peu d'existence à leurs yeux. De nombreux représentants syndicaux s'avèrent d'ailleurs surpris d'apprendre que les journalistes auxquels ils sont parfois confrontés ou risquent d'être confrontés relèvent le plus souvent de la catégorie « intellectuels précaires ». Cette image négative des journalistes, les craintes et les doutes nourris à l'égard des médias témoignent d'une méconnaissance de cet univers social qui n'est, le plus souvent, entrevu que par la lorgnette du petit écran. Ils traduisent plus sûrement encore la certitude ${ }^{9}$ que le regard porté sur le monde du travail sera de toute manière dépréciatif, que les médias ne peuvent pas être des relais servant leur cause. Ils constituent de ce fait un obstacle pour la médiatisation de toute action publique dans la mesure où de

\footnotetext{
9 À bon droit, on peut penser que le regard dépréciatif porté sur les médias est fondé si l'on se reporte à l'occultation du monde ouvrier et, plus généralement, du monde du travail au sein des fictions et, d'une manière plus complexe, au sein de l'information télévisée. Et l'on se gardera d'évoquer toutes les formes de stigmatisation dont ces êtres d'en bas sont affublés quand les médias daignent les évoquer.
} 
nombreux acteurs du monde syndical s'en tiennent à leurs préjugés sans trop chercher à travailler avec des journalistes susceptibles de constituer d'utiles relais en situation de conflits ou de crise. On imagine mal un quelconque délégué syndical développer par lui-même une gestion des « relations-presse » comparable à celle d'un directeur de la communication, voire d'un attaché de presse, mais on peut néanmoins s'étonner de l'absence de réflexion stratégique sur ce thème au sein des unions locales, départementales ou régionales, mais aussi des fédérations, voire des confédérations. La prudence à l'égard du monde des médias, pressenti comme étranger sinon hostile, témoigne d'une difficile prise en compte de la médiatisation comme terrain stratégique d'intervention pour valoriser et légitimer publiquement son action et, par répercussion, pour lutter en interne. Les représentants syndicaux se privent ainsi d'un outil d'action, sachant qu'à l'heure de la RSE (Responsabilité sociale des entreprises), les directions se montrent pourtant très sensibles à toute médiatisation mettant en jeu l'image de l'entreprise sur la place publique sous un jour potentiellement négatif. Le phénomène s'explique une nouvelle fois par une double absence de ressources: absences de ressources matérielles si l'on considère que tout développement d'une politique de communication suppose l'engagement de moyens, mais aussi absence de ressources symboliques au sens où le monde des médias paraît souvent inaccessible socialement et symboliquement à ces acteurs car il est, à sa manière, à l'image de la domination symbolique qu'ils subissent.

À l'échelon national, confédéral, la problématique se pose en des termes différents. II est vrai, si l'on en juge par la manière pour le moins condescendante dont un célèbre présentateur de JT, en l'occurrence David Pujadas ${ }^{10}$, peut s'adresser à des représentants syndicaux, que les grands médias nationaux sont peu favorables à certaines organisations syndicales. Dans le cas particulier de l'interview de Xavier Mathieu, DS CGT de l'entreprise Continental, ce n'est rien d'autre que le mépris du monde ouvrier qui transparaissait ouvertement. Confronté à un individu de cette classe, le journaliste pouvait s'ériger inconsciemment en qualité de porte-parole de son groupe social et manifester qu'en raison de l'origine et du statut de son interlocuteur, il lui était difficile d'échanger civilement, de s'adresser à lui d'égal à égal, pour ne pas dire comme à un citoyen, trahissant ainsi d'inavouables préjugés politiques et sociaux. Révélateur à ce titre, ce cas est cependant exceptionnel en raison de la dissymétrie sociale des protagonistes. Les luttes sociales ne sont certes pas très médiatiques, en particulier dans leur versant « lutte », comme si la mise au jour de la conflictualité au sein du monde du travail rappelait cette insupportable vérité de la division sociale du travail et des injustices qui en résultent. Mais en règle générale, dans des phases de jeu médiatique plus routinière, associant des partenaires habitués les uns aux

10 On se souvient de la réaction offusquée de J.-L. Mélenchon devant l'image du présentateur du JT de France 2, D. Pujadas, interpellant X. Mathieu (délégué syndical CGT - Continental) au moment où le conflit atteignait son paroxysme, en lui demandant sur un ton réprobateur et paternaliste : « Est-ce que vous regrettez ? » (extrait du documentaire de P. Carles, Fin de concession. Accès : http://www.youtube.com/watch?v=aDX_jVs7mPQ). 
autres (journalistes de la presse audiovisuelle nationale/membres dirigeants des confédérations ou des fédérations) les échanges tendent à s'équilibrer au travers de jeux bien rôdées au sein desquels chacun respecte le registre d'intervention de l'autre. Pour les confédérations et les acteurs nationaux (secrétaires généraux), les problèmes résident donc moins dans les relations personnalisées aux médias et aux journalistes en raison des phénomènes de routinisation des relations, y compris dans les situations de crise, que dans la confrontation à des logiques médiatiques qu'ils ne parviennent pas à maîtriser. L'enjeu majeur pour les confédérations est d'influer sur l'agenda des médias en maîtrisant un agenda social façonné par eux, en imposant ainsi leur temporalité. La difficulté s'avère double. Elle découle d'abord du morcellement du paysage syndical et des divisions internes aux confédérations qui ne facilitent pas les phénomènes de mobilisation collective et neutralisent la possibilité d'agir rapidement et de manière efficace pour imposer son tempo. Elle renvoie ensuite à la facilité avec laquelle d'autres acteurs, politiques et publics notamment, peuvent modifier un agenda construit de longue haleine du simple fait de leur capacité à faire l'actualité et à l'imposer aux médias. Une étude systématique reste à entreprendre sur ce point pour mesurer avec plus de précision le déficit stratégique dans la relation aux médias dont pâtissent les organisations syndicales qui subissent l'agenda médiatique et politique et dont les stratégies, en raison de leur inertie institutionnelle, sont trop lisibles aux yeux des partenaires de jeu pour ne pas être endigué.

Cette inertie découle de la difficulté des confédérations à se positionner en porte-parole légitime à l'égard de leurs propres composantes (fédérations, unions, syndicats, etc.). Les prises de parole des secrétaires confédéraux témoignent souvent de ces tensions qui conduisent à l'expression de positions particulièrement policées, témoignant de trop savants jeux d'équilibre. Quelques envolées lyriques sur l'euro, la crise monétaire et/ou la mondialisation, qui ne sont pas sans évoquer les prises de parole de leader politique, dissimulent mal ces problèmes. On peut s'interroger sur la perception - par les militants, les adhérents, les sympathisants, les simples salariés - de ces prises de position qui renvoient l'action syndicale dans les hautes sphères de la science médiaticopolitique. À ce titre, la communication des confédérations s'apparente plus souvent à un symptôme loquace des problèmes de compatibilité interne et de positionnement entre confédérations qu'à l'expression d'une politique clairement définie répondant à des enjeux précis pour les salariés.

Dernier point. La personnalisation du message syndical sert et dessert les organisations. Pour une grande partie des salariés, pour les journalistes, pour des observateurs peu avertis, la CGT, la CFDT, la CGT-FO... se résument aux points de vue confédéraux qu'expriment leurs porte-parole. II en résulte une focalisation sur la personnalité des secrétaires confédéraux et la croyance selon laquelle ce qu'ils disent est l'expression de ce que pensent les membres de l'organisation. Outre la méconnaissance de l'hétérogénéité des confédérations et du caractère mouvant de leur relief, il en résulte une sorte de culte de la personnalité qui 
convient parfaitement au monde des médias soucieux de promouvoir quelques bons clients afin de s'assurer une audience mais qui comporte quelques risques pour l'expression syndicale. À lui seul, le cas de Marc Blondel (FO) mériterait une monographie pour préciser jusqu'à quel point les acteurs peuvent jouer/déjouer les stratégies journalistiques de médiatisation mais aussi quels sont les limites et les risques d'un tel jeu qui revient à effacer le message syndical derrière le spectacle d'une personnalité dont les journalistes se servent et qu'ils desservent.

\section{Conclusion}

Dans sa dénonciation de la faible utilisation des TIC comme moyen de mobilisation, le journaliste évoqué en introduction n'avait pas totalement tort. On peut regretter que les TIC, les réseaux sociaux et autres signes de modernité ne soient pas davantage investis par les principales organisations syndicales. En pratique, qu'en est-il ? On peut d'abord observer que les sites des organisations syndicales, des confédérations en premier lieu mais aussi de fédérations ou d'unions mettent à la disposition des sympathisants, des adhérents, des militants, des ressources relativement abondantes en informations. À ce titre, supposer qu'il y a « sous-utilisation » appelle de plus amples analyses et, surtout, un regard plus attentif aux stratégies d'usage. Le délégué syndical ne peut en effet recourir à l'intranet de l'entreprise et diffuser de l'information en dehors d'un accord écrit avec la direction qui en définit et en restreint les modalités. II peut certes créer librement un site, un blog ou constituer par lui-même une liste de diffusion s'adressant aux salariés lui ayant préalablement communiqué leur adresse privée mais ses moyens s'avèrent de ce fait limités. II lui faut disposer matériellement du temps, techniquement de la compétence, physiquement de l'énergie pour que le blog ou la gestion d'une mailing-liste bénéficie d'une audience significative chez les salariés. Encore faut-il que ceux-ci soient réceptifs techniquement et syndicalement. On comprend pourquoi certains observateurs perçoivent une sous-utilisation qui n'est, en fait, qu'une utilisation pratiquement limitée des TIC dans le cadre de l'action syndicale. L'erreur consisterait donc à spéculer sur d'hypothétiques non-usages et sur leurs non moins hypothétiques causes. Non seulement cela traduirait un penchant à l'intellectuallisation incontrôlée que de supposer une forme quelconque de résistance consciente ou inconsciente à l'usage des TIC parce qu'elles seraient les vecteurs de valeurs ou de mythes, mais ce serait se méprendre sur les velléités d'appropriation et d'usage des militants syndicaux et, surtout, sur les limites matérielles rencontrées par ces même acteurs, confrontés à des difficultés inhérentes à leur mode de vie, à leurs ressources et, plus encore, à leur socialisation professionnelle.Voir le monde avec des lunettes de journalistes qui passent une grande partie de la journée le regard rivé à un écran d'ordinateur conduit à croire que les mobilisations sociales via Facebook produisent des grèves voire des révolutions sociales. Autre illusion : croire que les salariés qui travaillent en usine ou dans la distribution ou dans 
tout autre secteur ont le temps de consacrer quelques heures à surfer sur le net, à consulter des blogs, à entretenir des amitiés réticulaires en multipliant les messages. Outre l'absence de temps, ces activités nécessitent un certain nombre de compétences qui peuvent paraître élémentaires mais dont sont dépourvus un nombre significatif de salariés. Dès lors, en supposant qu'un représentant syndical dispose de l'énergie, des moyens matériels et du temps, pour développer une communication au moyen de ce type de support, que peut-il en être de la réception de ses performances communicationnelles par un public pour le moins peu réceptif?

Peut-être objectera-t-on ici que les nouvelles générations de salariés manifesteront une plus grande propension à recourir à ce type d'outils et d'informations, comme en témoigne les usages de certains groupes de militants radicaux, comme en témoigne également l'engouement des plus jeunes pour toutes les formes de communication médiatisées (téléphonie mobile, réseaux sociaux, etc.). Rien ne prouve que cet engouement puisse trouver une traduction en matière d'action syndicale pour la simple raison que ces nouvelles formes sociales de rapports médiatisés s'avèrent éloignées des logiques de la communication et de l'action syndicale. II reste par conséquent à imaginer ce que pourraient être de nouveaux types d'usage de ces technologies qui soient à la fois adaptés aux besoins de l'action syndicale en entreprise et conformes aux caractéristiques sociales de la population des salariés. La connaissance des usages TIC au sein des organisations conduirait à porter un regard prudent car ces technologies n'échappent pas, comme on l'oublie trop souvent à force de généralisation, à des logiques de différenciation sociale qu'elles tendent à démultiplier, non pas en raison de leur nature perverse, mais parce qu'elles profitent d'abord à ceux qui disposent des ressources pour en tirer profit.

\section{Références}

Andolfatto D., Labbé D., 2007, Sociologie des syndicats, Paris, Éd. La Découverte.

— 2009, Toujours moins! Dédlin du syndicalisme à la française, Paris, Gallimard.

Beaud S., Pialoux M., 1999, Retour sur la condition ouvrière, Paris, Fayard.

Bourdieu P, 1973, «L'opinion publique n'existe pas », pp. 222-235, in : Questions de sociologie, Paris, Éd. de Minuit, 1984.

— 1977, Sur le pouvoir symbolique, Annales. Économies, Sociétés, Civilisations, 32, 3, pp. 405411 .

CFDT, 1994, Guide de la communication syndicale, Paris, CFDT.

Contrepois S., 2003, Syndicats. La nouvelle donne, Paris, Éd. Syllepse.

François B., Neveu É., dirs, 1999, Espaces publics mosaïques, Acteurs, arènes et rhétoriques des débats publics contemporains, Rennes, Presses universitaires de Rennes.

ISERES, 2000, « Syndicalisme et communication », Les documents de l'ISERES, Paris, CGT. 
Lehingue P., 2007, Subunda. Coups de sonde dans l'océan des sondages, Paris, Éd. du Croquant.

Lévêque S., 2000, Les journalistes sociaux. Histoire et sociologie d'une spécialité journalistique, Rennes, Presses universitaires de Rennes.

Mouriaux R., 1998, Crises du syndicalisme français, Paris, Montchrestien.

Neveu É., 2005, Sociologie des mouvements sociaux, Paris, Éd. La Découverte.

Olivesi S., 200 I, « Les PME face aux 35 h. Réduction du temps de travail, négociation sociale et dispositif "appui-conseil" », La Pensée, 328, pp. 77-99.

- 2011, « La formation en communication des représentants syndicaux. Logiques institutionnelles et enjeux militants », Réseaux, 170, pp. 163-189.

- à paraitre, Observer l'autoscopie. Les représentants syndicaux dans "la société de communication $》$.

Piotet F., dir., 2009a, La CGT et la recomposition syndicale, Paris, Presses universitaires de France.

— 2009b, « La CGT, une anarchie (plus ou moins) organisée ? », Politix, vol. 22, 85, pp. 9-30.

Rosanvallon P., 1988, La question syndicale, Paris, Calmann-Lévy.

Sainsaulieu R., dir., 1990, L'Entreprise, une affaire de société, Paris, PFNSP.

— 1994, Méthodes pour une sociologie de l'entreprise, Paris, PFNSP/ANACT.

Salem A., 1993, « De travailleurs à salariés. Repères pour une étude de l'évolution du vocabulaire syndical ( 197 I - 1990) », Mots. Les langages du politique, vol. 36, I, pp. 74-83.

Schwartz O., 1990 Le monde privé des ouvriers. Hommes et femmes du Nord, Paris, Presses universitaires de France.

Terrail J.-P., 1990 Destins ouvriers. La fin d'une classe?., Paris, Presses universitaires de France.

Ubbiali G., 1993 « Syndicalisme en crise, syndicalisme en mutation. Note critique », Politix, vol. 6, 23, pp. 84-।0I.

Willemez L., 2003/4, « Quand les syndicats se saisissent du droit. Invention et redéfinition d'un rôle », Sociétés contemporaines, 52, pp. 17-38. 